Supplement of Biogeosciences, 17, 1655-1672, 2020

https://doi.org/10.5194/bg-17-1655-2020-supplement

(C) Author(s) 2020. This work is distributed under

the Creative Commons Attribution 4.0 License.

(c) (1)

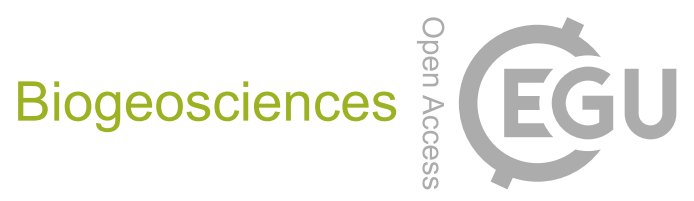

Supplement of

\title{
Quantifying impacts of the 2018 drought on European ecosystems in comparison to 2003
}

\author{
Allan Buras et al. \\ Correspondence to: Allan Buras (allan@buras.eu)
}

The copyright of individual parts of the supplement might differ from the CC BY 4.0 License. 

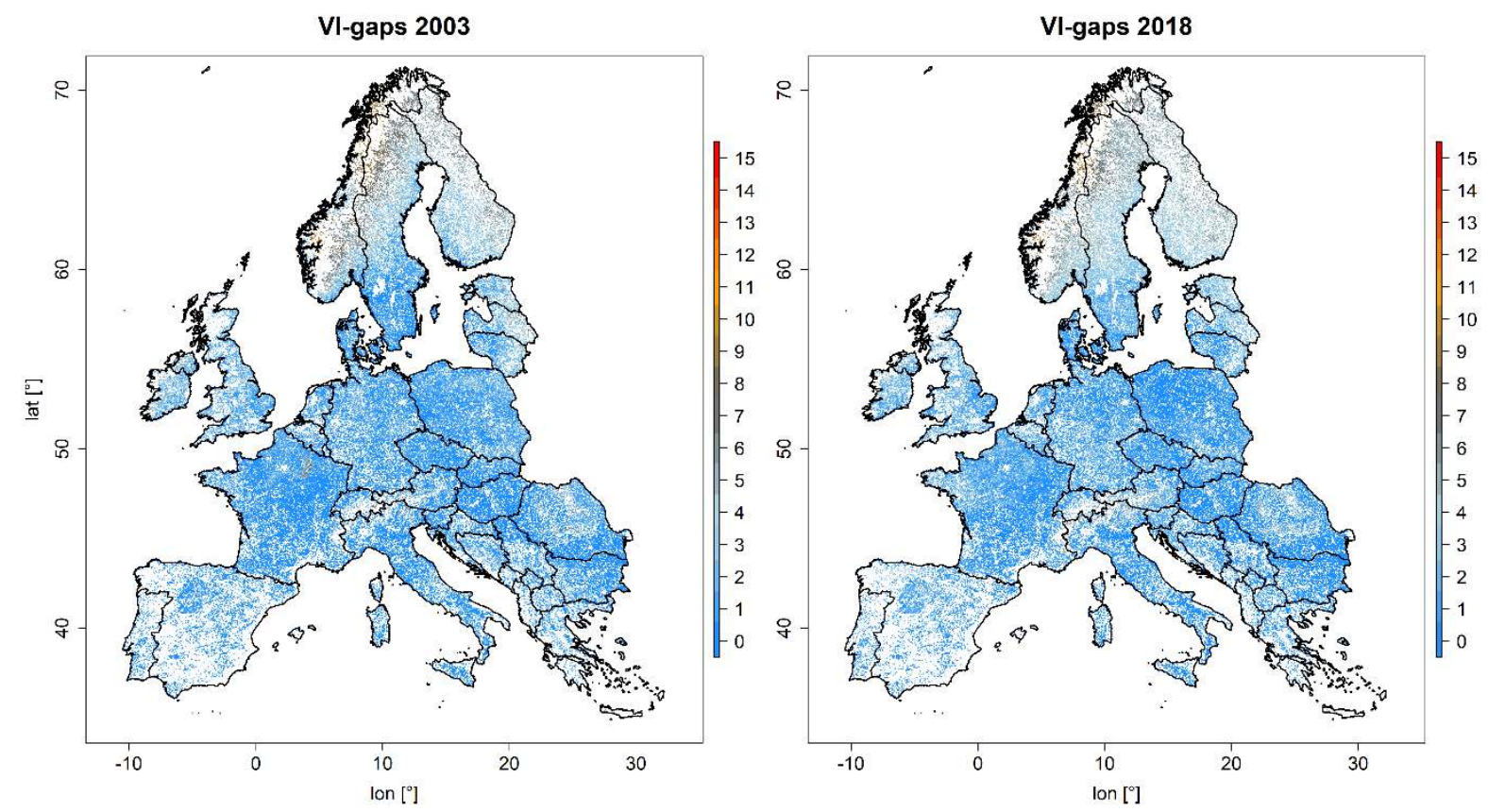

Fig. S1: Number of gaps for the MODIS vegetation indices (VI) that were filled using linear interpolation during the data pre-processing for 2003 (a) and 2018 (b) (for details see section 2.1.2).

(a) NDVI linear trend $2000-2018$

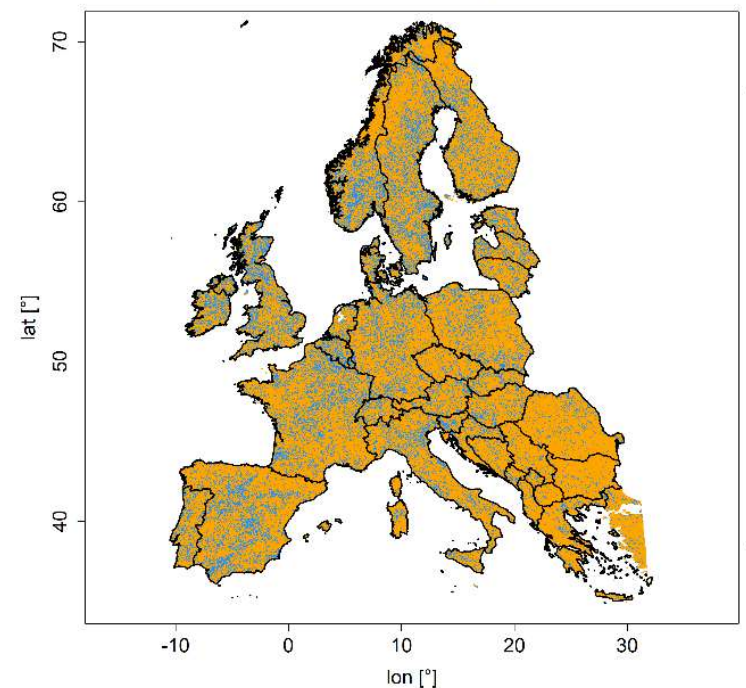

(b) EVI linear trend 2000-2018

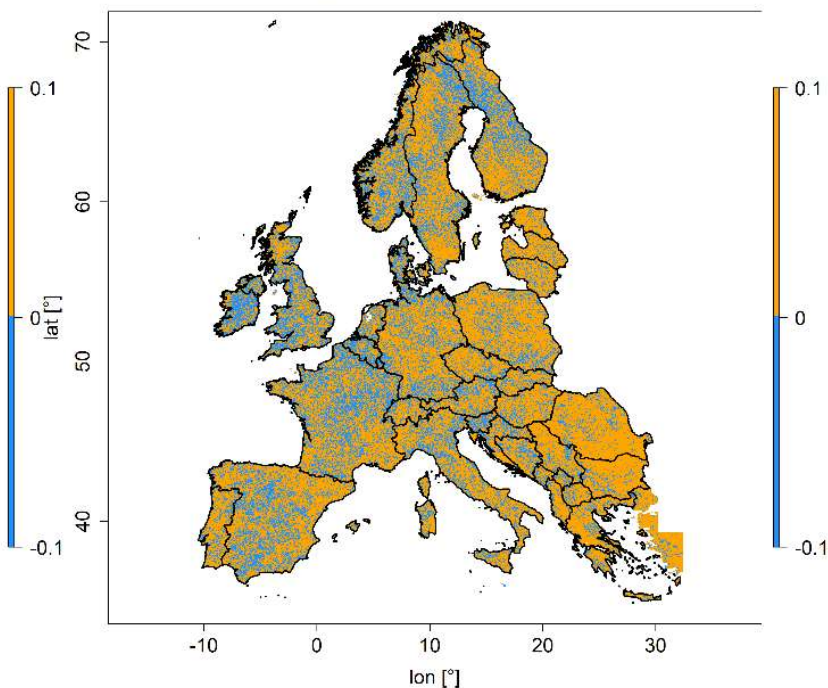

Figure S2: Maps depicting temporal trends of NDVI (a) and EVI (b) over the whole study period. Individual trends were subtracted from each individual pixel time-series. 


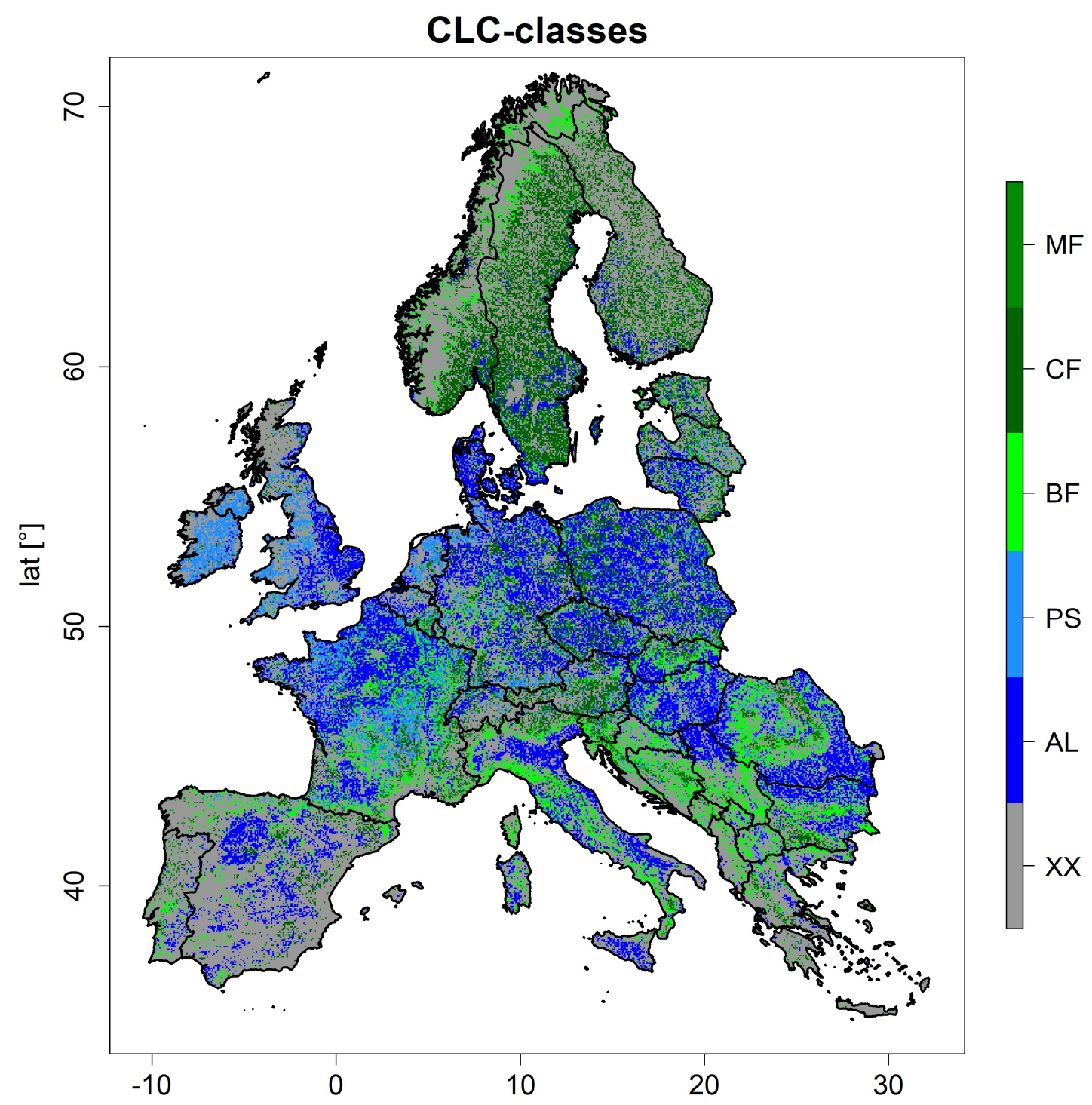

Fig. S3: Map depicting the MODIS pixels that were used in our analyses as well as their corresponding CLC land-cover calls. $\mathrm{XX}=$ masked pixels, $\mathrm{AL}=$ arable land, $\mathrm{PS}=$ pastures, $\mathrm{BF}=$ broadleaved forest, $\mathrm{CF}=$ coniferous forest, $\mathrm{MF}=$ mixed forest.
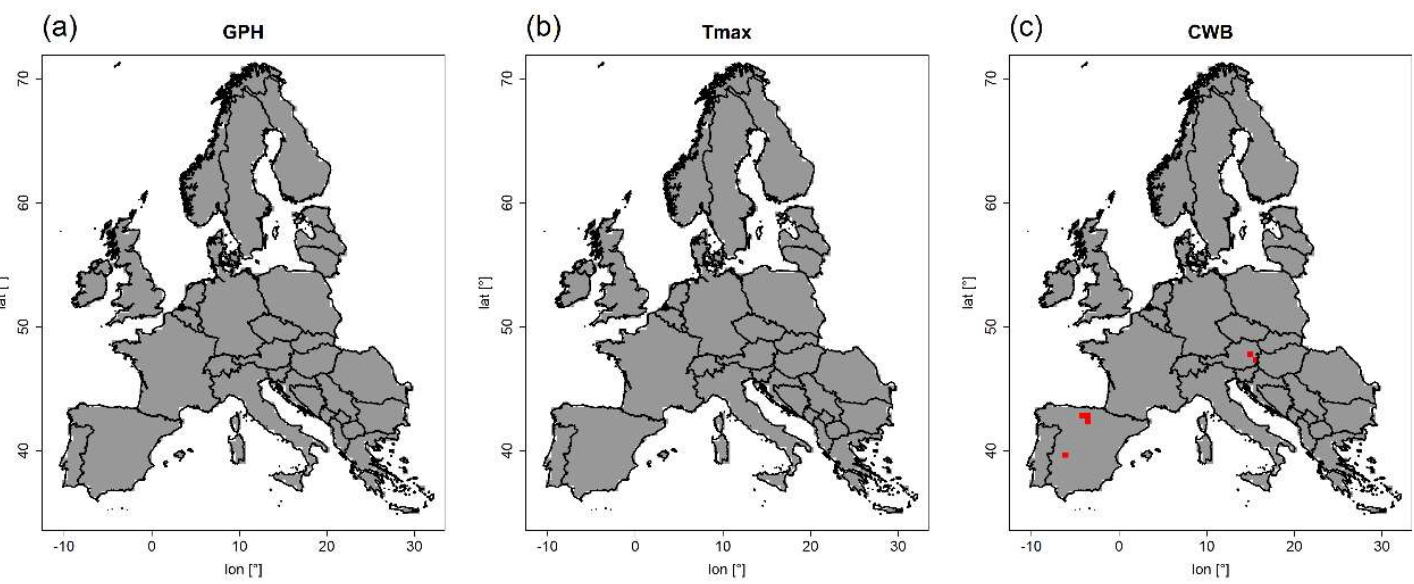

Figure S4: Maps depicting significance of Shapiro-Wilk normality test (red pixels refer to $p<0.001$ ) for 500 hPa geopotential height (a), Tmax (b), and climatic water balance (c). The number of significant tests ranges from 0 percent for geopotential height to 0.1 percent for climatic water balance and thus lies within the order of expected type I errors ( 0.1 percent). 
Tmax anomalies
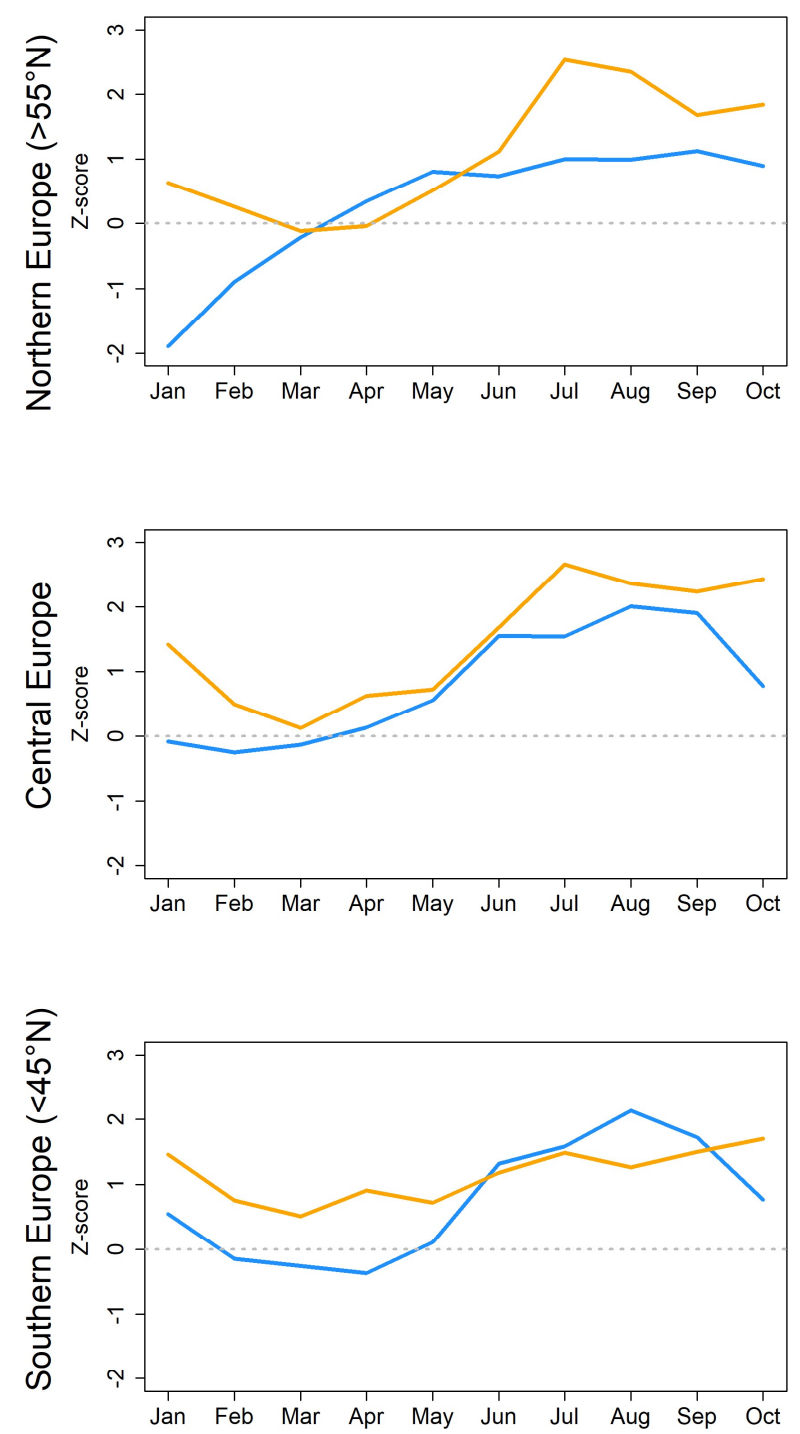

CWB anomalies
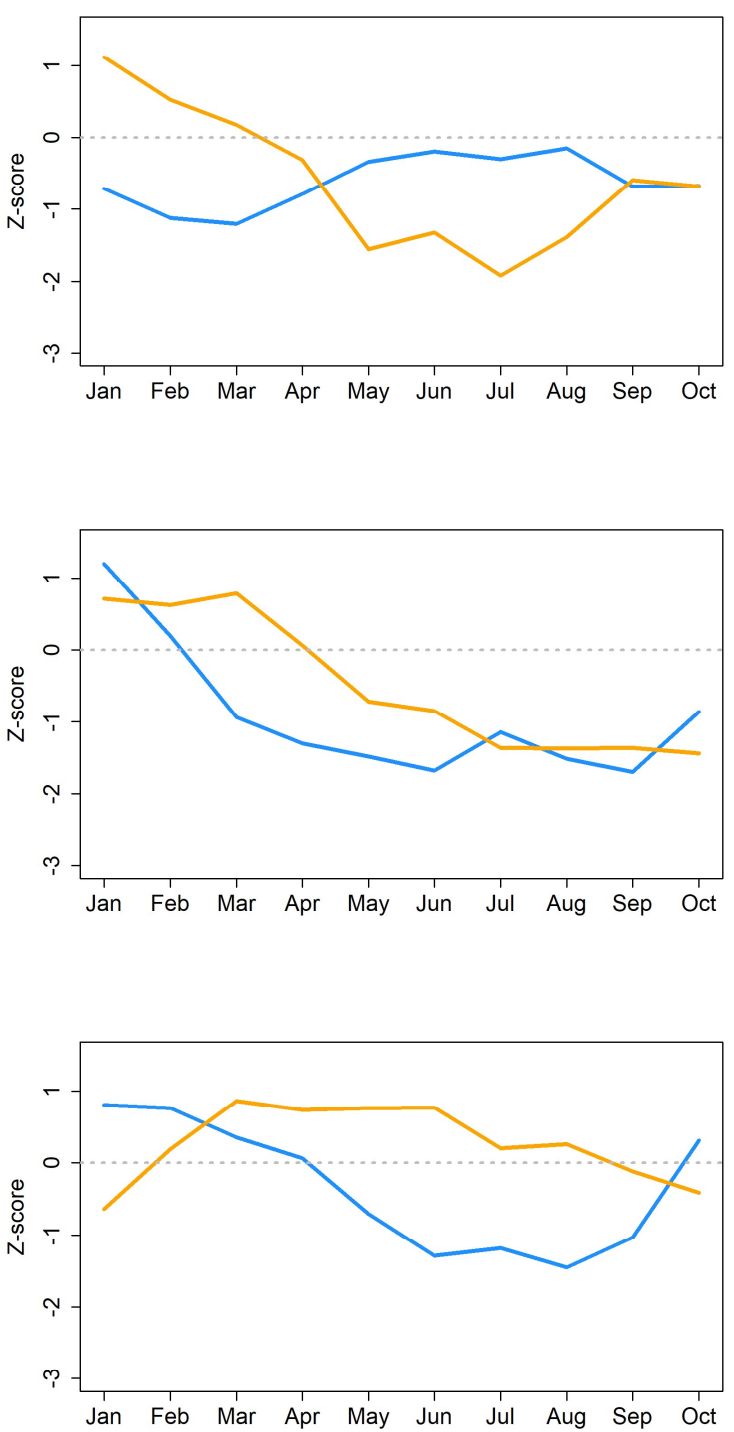

Fig. S5: Time series depicting the temporal development of Tmax anomalies (left panels) and climatic water balance (right panels) for Northern (upper panels), Central (mid panels) and Southern (lower panels) Europe. Blue refers to 2003, orange to 2018. 


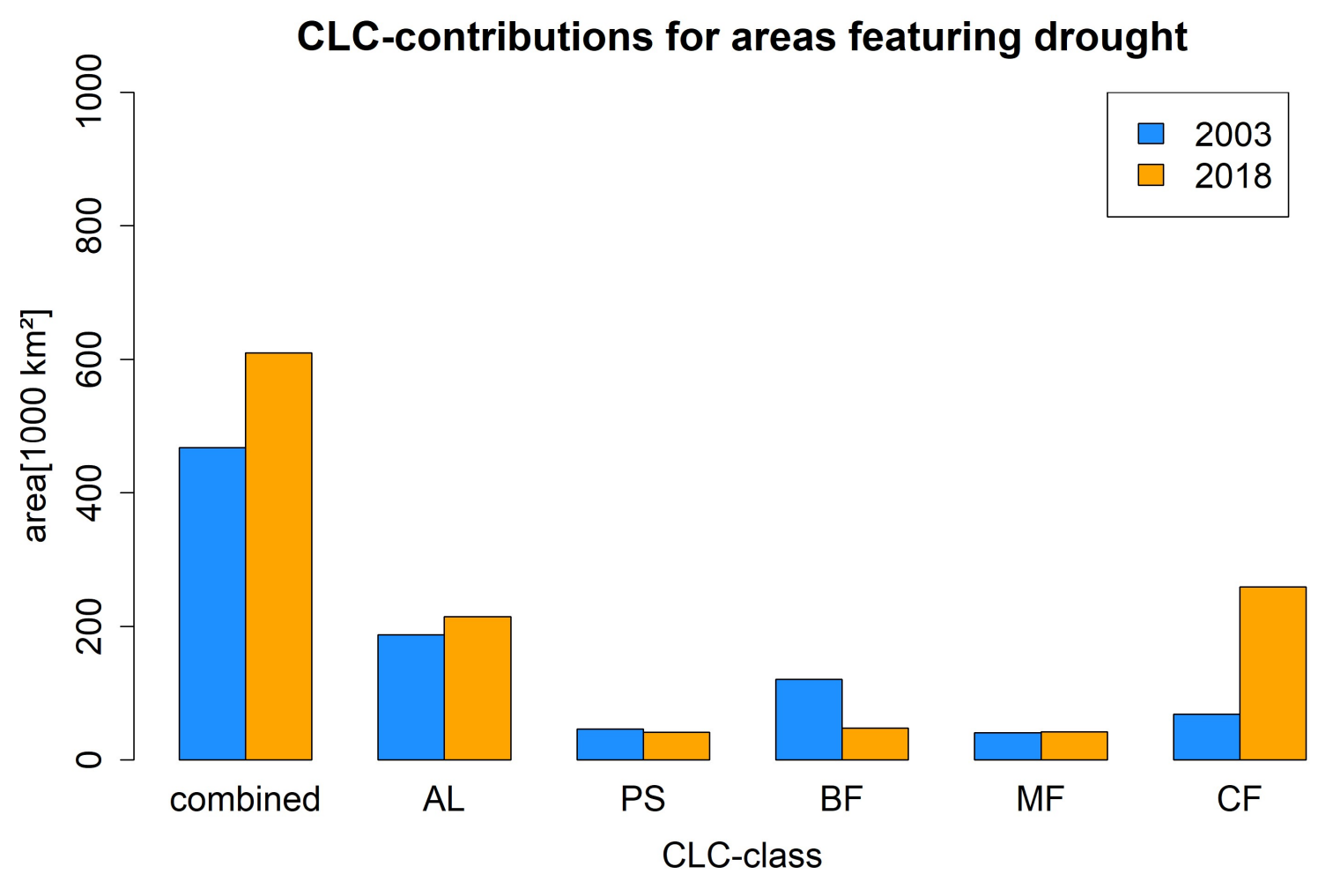

Fig. S6: Barplot depicting the absolute share of Corine land cover classes to the regions featuring extreme water deficit (CWB anomalies $<-2)$. $A L=$ arable land, $P S=$ pastures, $B F=$ broadleaved forest, $\mathrm{MF}=$ mixed forest, $\mathrm{CF}=$ coniferous forest. The term combined refers to a combination of all five land cover classes. 

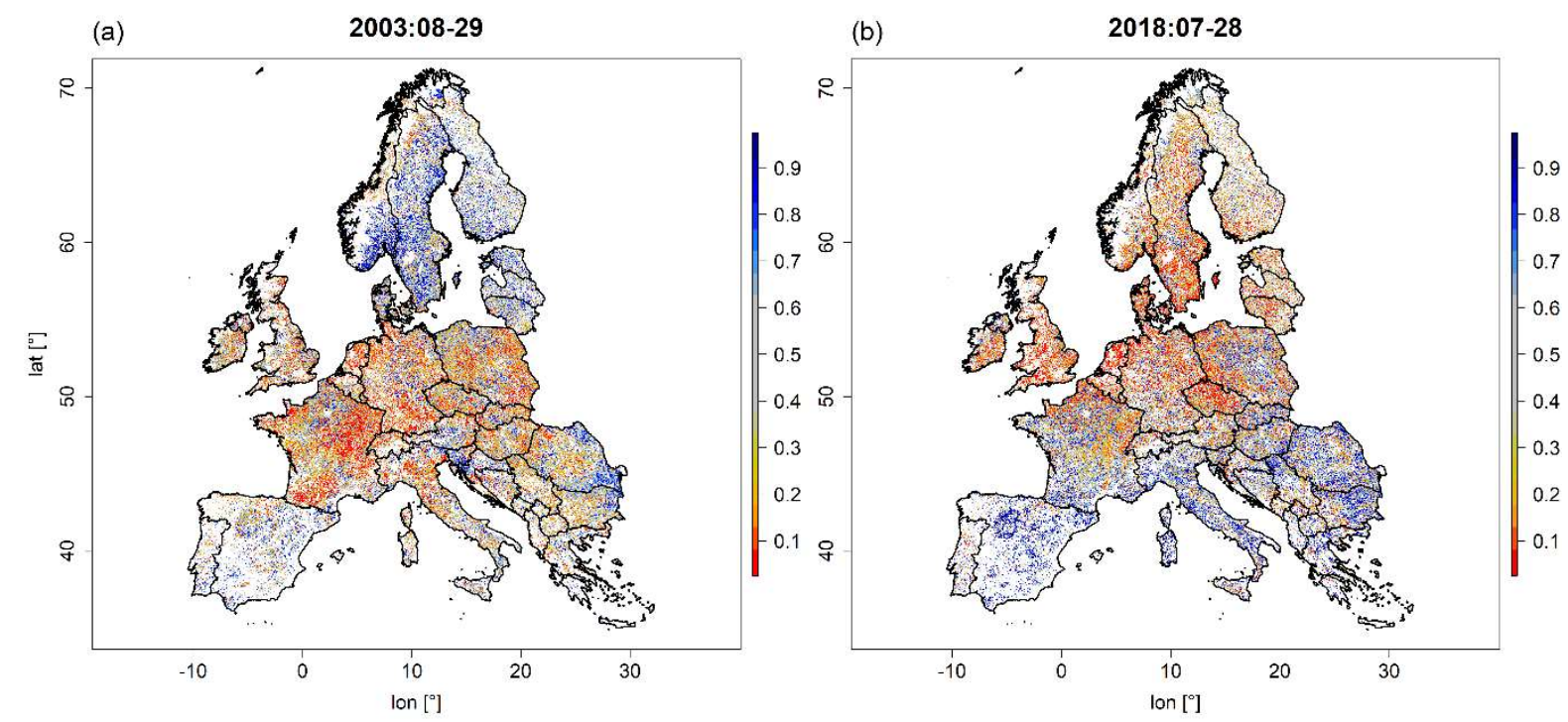

(1)

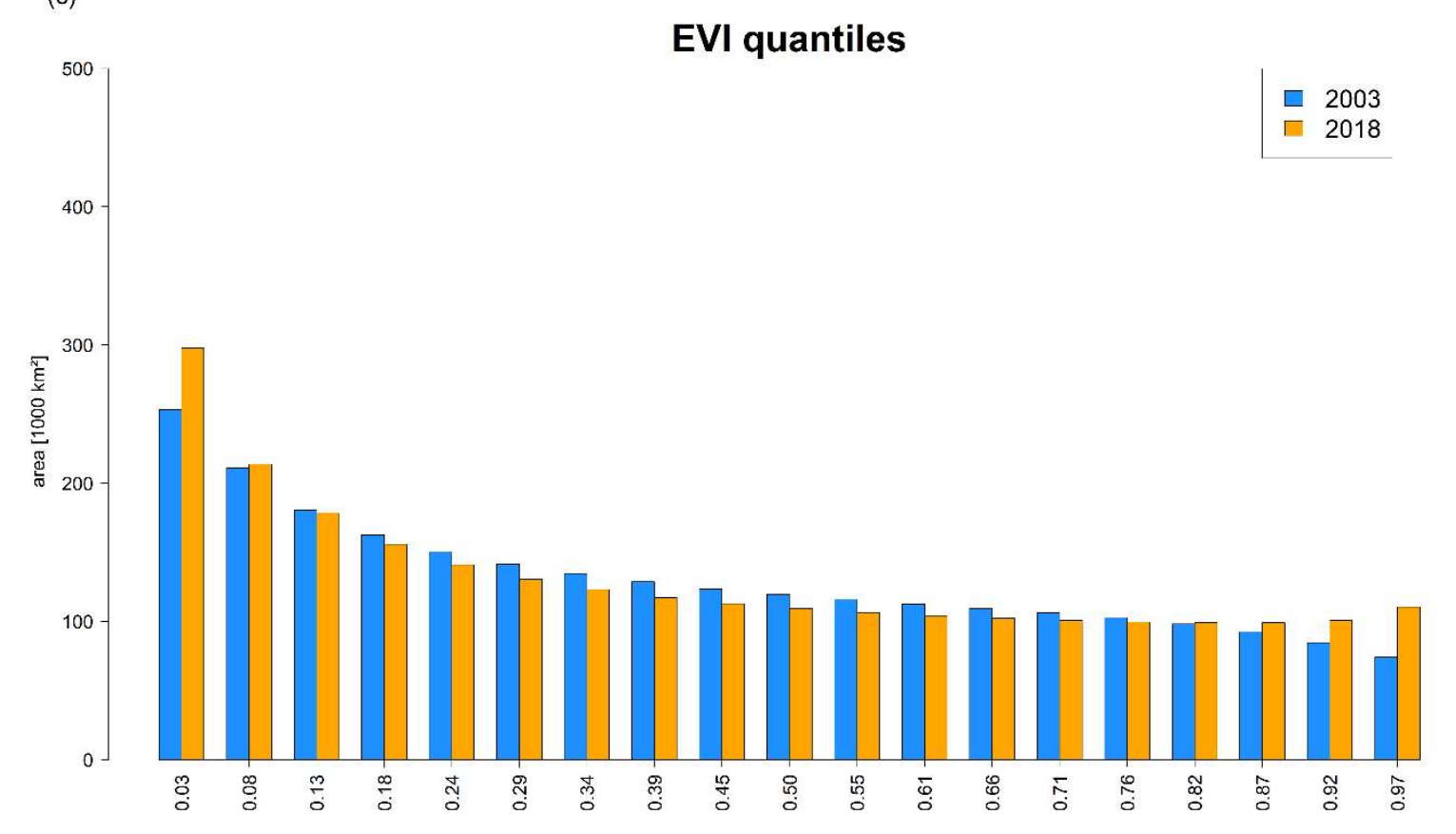

Fig. S7: MODIS EVI quantiles representing peak of drought conditions at the end of August (DOY 241) in 2003 (a) and end of July (DOY 209) in 2018 (b) for the five selected land-cover types as well as the corresponding area histograms (in units of $1000 \mathrm{~km}^{2}$ ) representing the nineteen EVI quantiles (c). Blue colors in (a) and (b) indicate upper quantiles (thus a higher than average vegetation greenness), while orange to red colors indicate lower anomalies (i.e. lower than average vegetation greenness). Blue bars in (c) refer to 2003 and orange bars to 2018. 
(a) CWB anomaly $<-2$
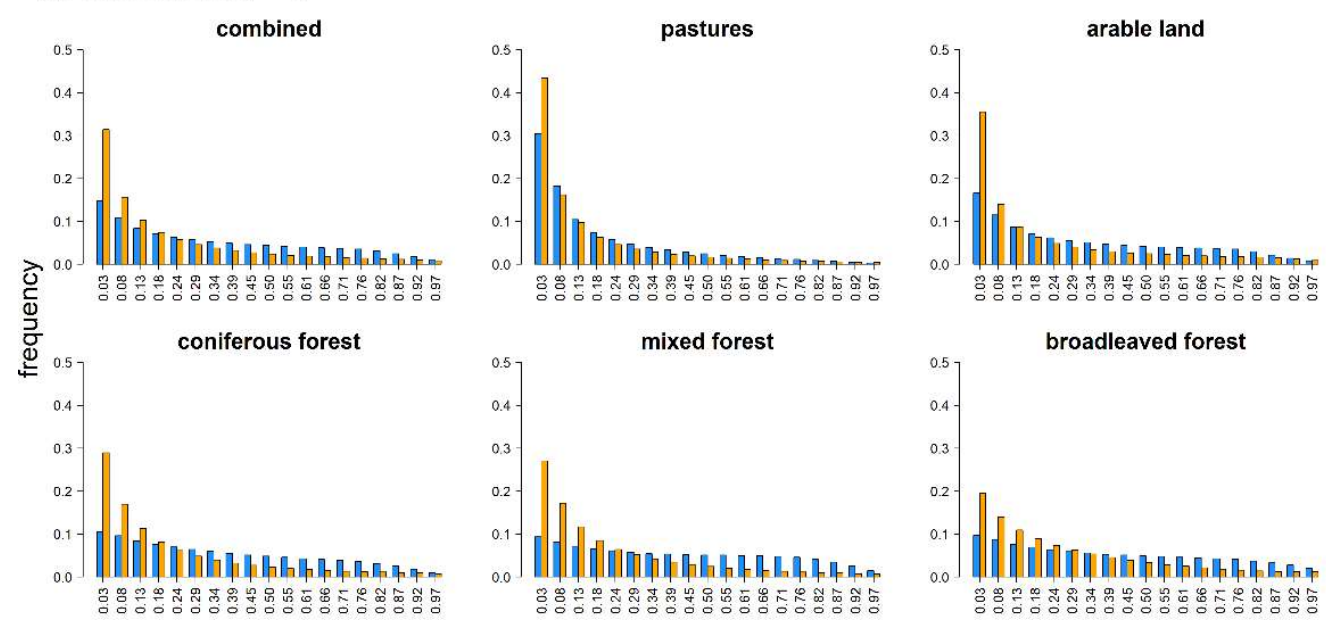

(b) $-2<$ CWB anomaly $<0$
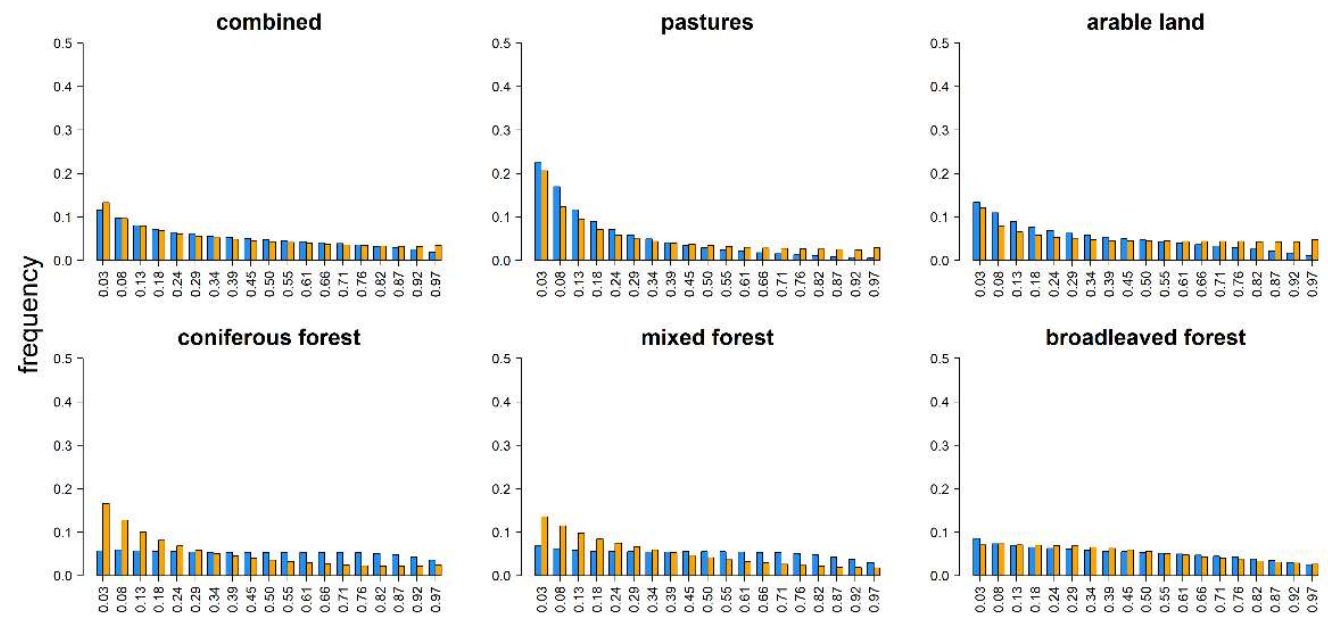

(b) CWB anomaly $>0$
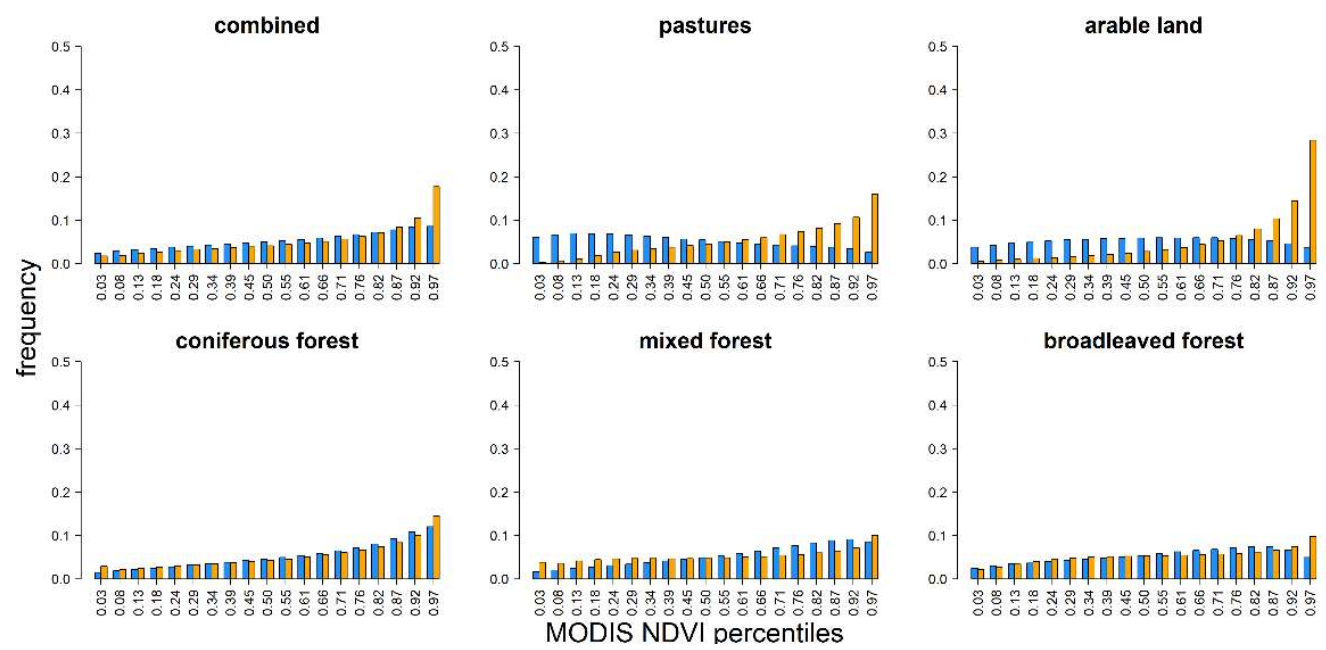

Fig. S8: Histograms depicting the proportions representing the nineteen NDVI quantiles pooled according to CORINE landcover classes for regions that featured (a) extreme water deficit (CWB-anomaly $<-2)$, (b) weak water deficit $(-2<\mathrm{CWB}$-anomaly $<0$ ), and (c) no water deficit (CWB-anomaly $>0$ ). Blue bars refer to 2003 , orange bars to 2018 . 
(a) CWB anomaly $<-2$
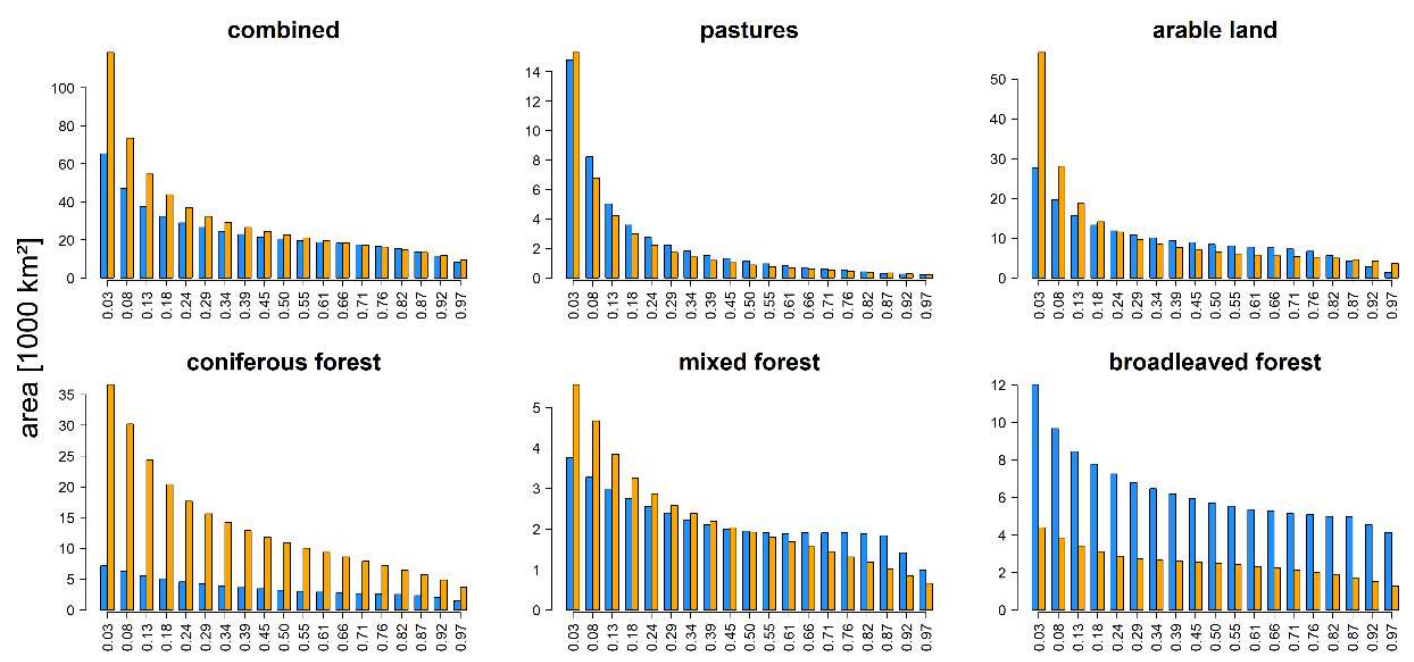

(b) $-2<$ CWB anomaly $<0$
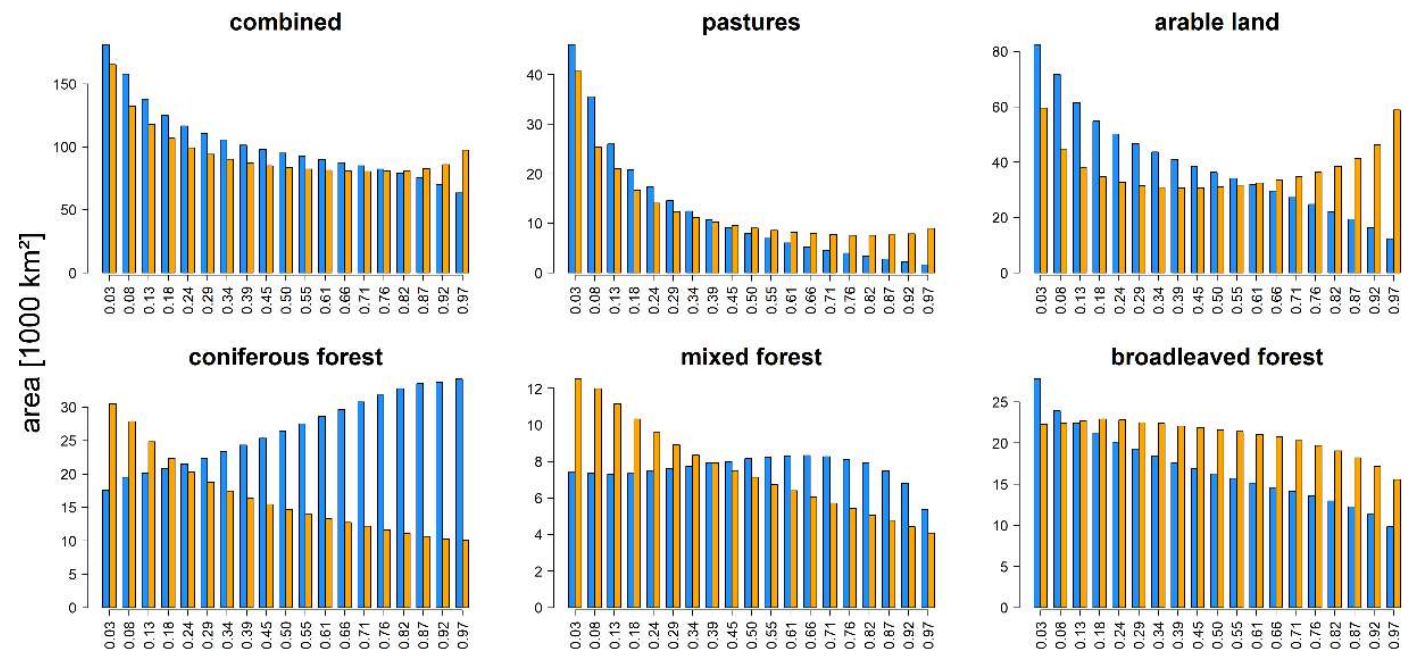

(c) CWB anomaly $>0$
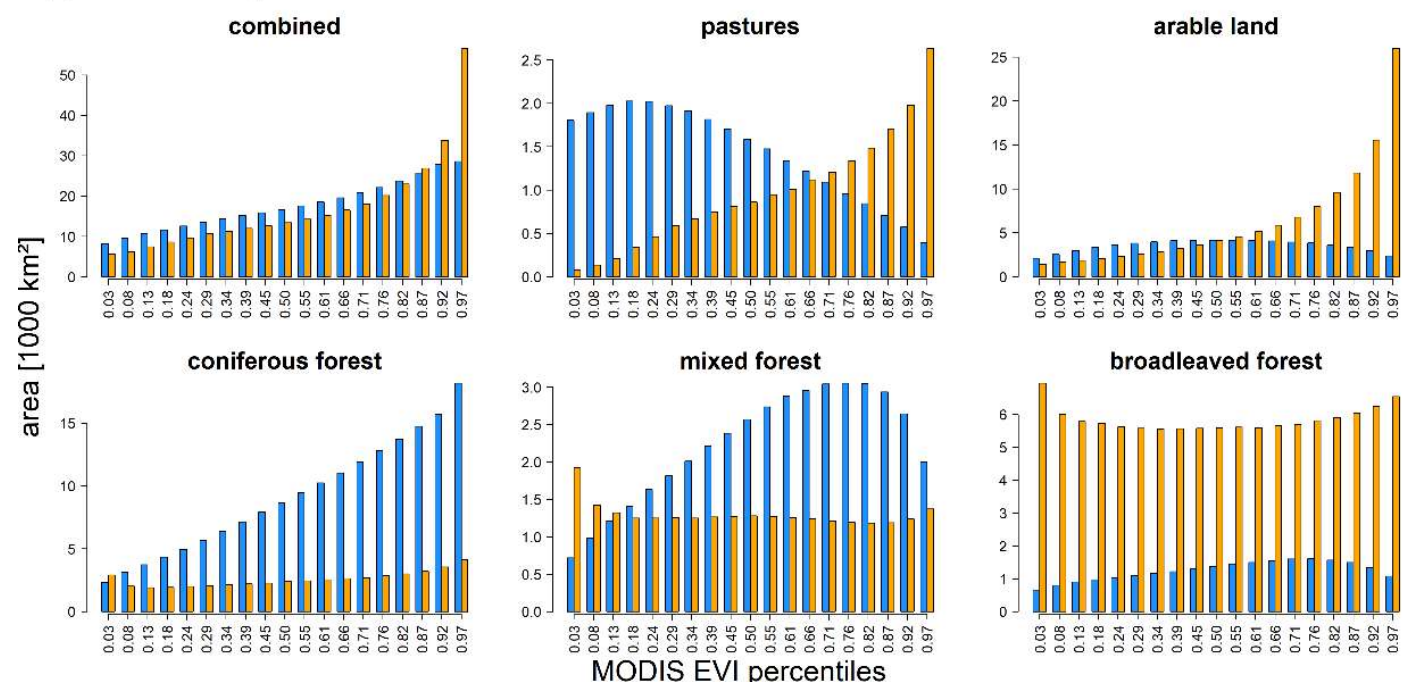

Fig. S9: Histograms depicting the absolute areas (in units of $1000 \mathbf{~ k m}^{2}$ ) representing the nineteen EVI quantiles pooled according to CORINE land-cover classes for regions that featured (a) extreme water deficit (CWB-anomaly < -2), (b) weak water deficit $(-2<$ CWB-anomaly $<0$ ), and (c) no water deficit (CWBanomaly $>0$ ). Blue bars refer to 2003, orange bars to 2018. Histograms depicting the proportional areas are shown in supplementary Fig. S10. 
(a) CWB anomaly $<-2$
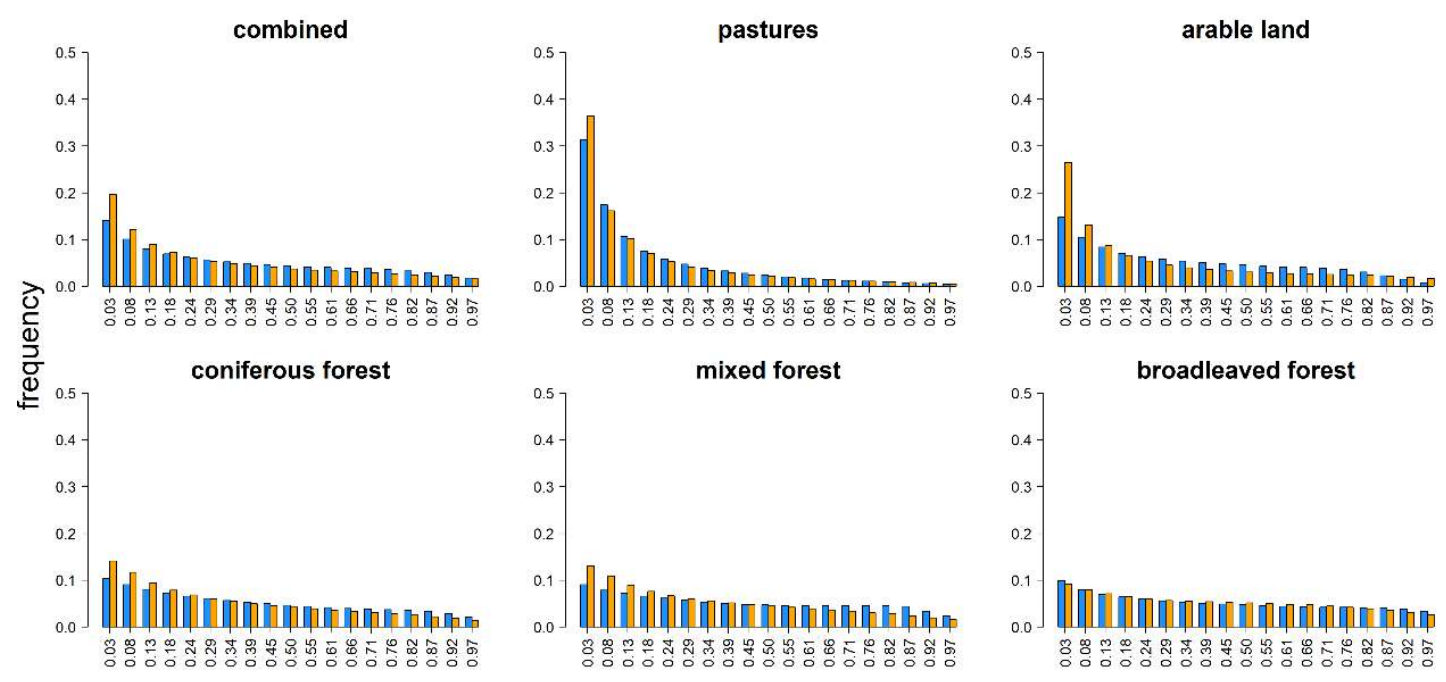

(b) $-2<$ CWB anomaly $<0$
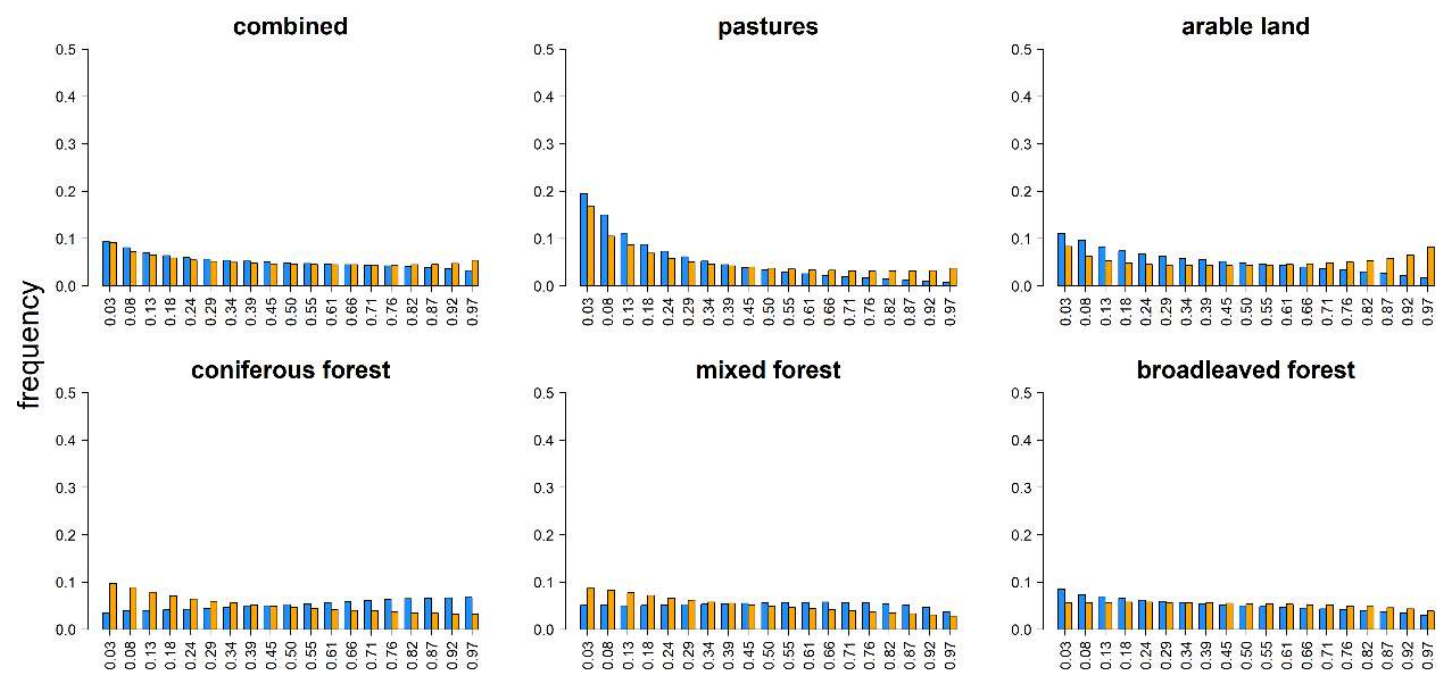

(b) CWB anomaly $>0$
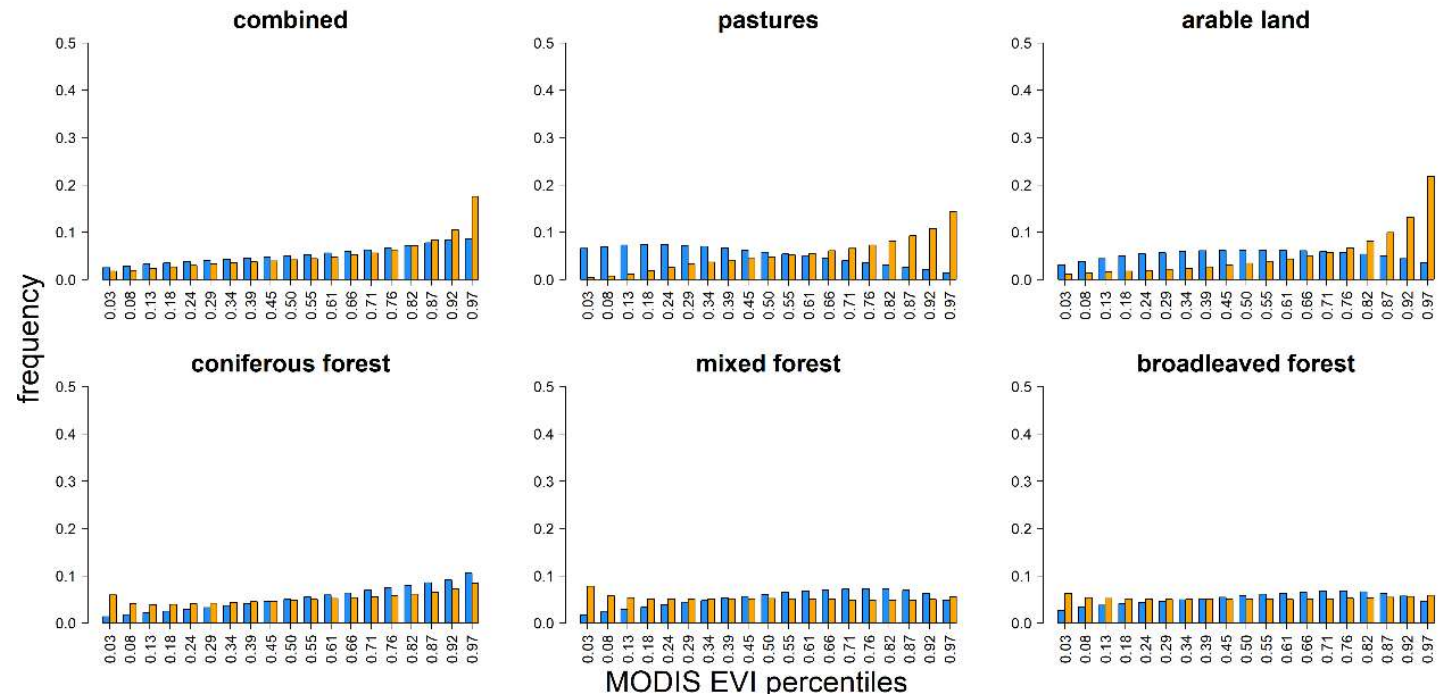

Fig. S10: Histograms depicting the proportions representing the nineteen EVI quantiles pooled according to CORINE landcover classes for regions that featured (a) extreme water deficit (CWB-anomaly $<-2)$, (b) weak water deficit $(-2<\mathrm{CWB}$-anomaly $<0$ ), and (c) no water deficit (CWB-anomaly $>0)$. Blue bars refer to 2003 , orange bars to 2018 . 


\section{CWB anomaly intersection}

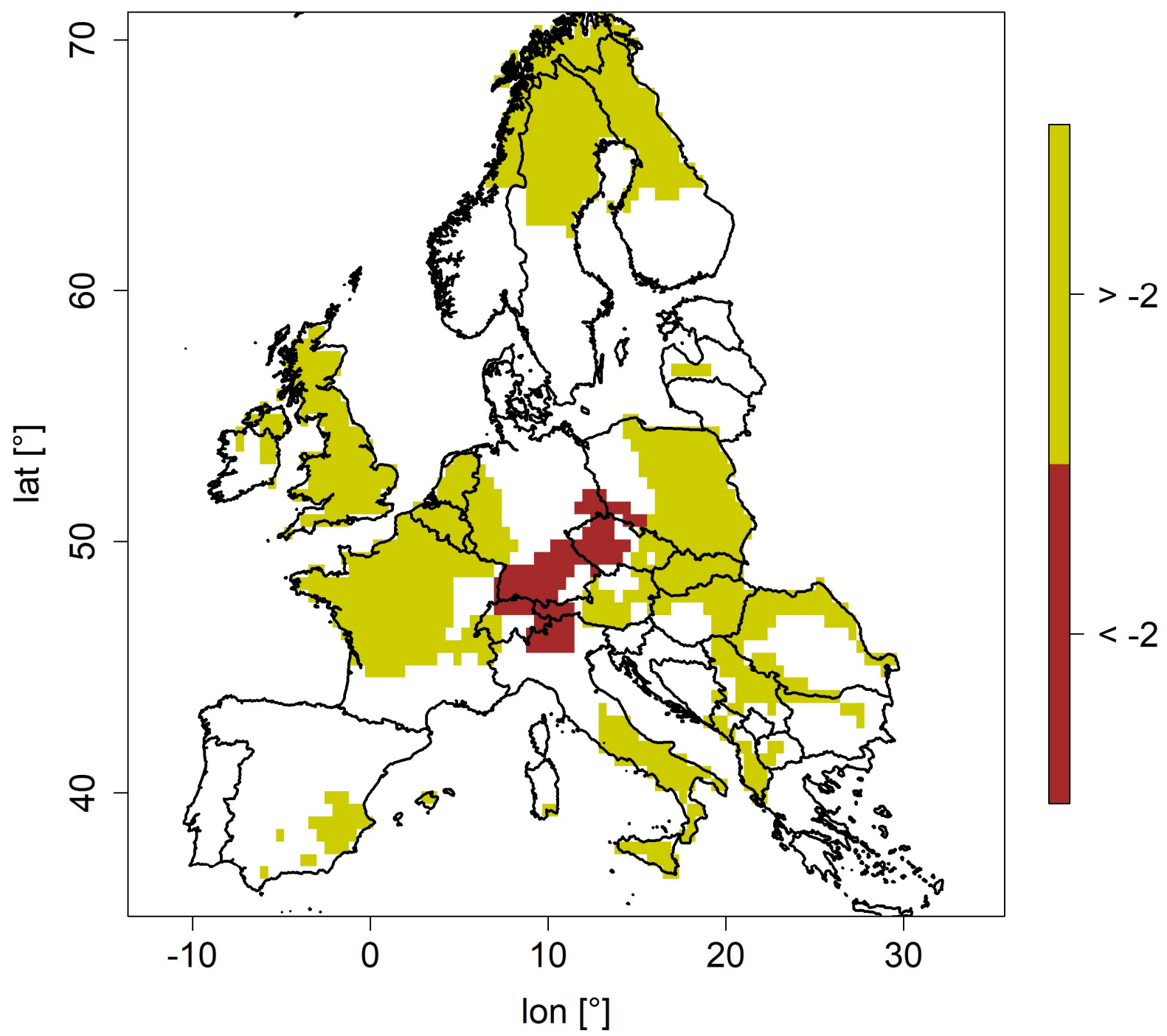

Fig. S11: Intersection areas of regions which featured extreme water deficit (CWB anomaly $<-2$, brown) and weak water deficit $(-2<C W B$ anomaly $<0$, yellow-green) in both 2003 and 2018 that were used for the analyses depicted in Figs. S12 and S13. 
(a) CWB anomaly $<-2$
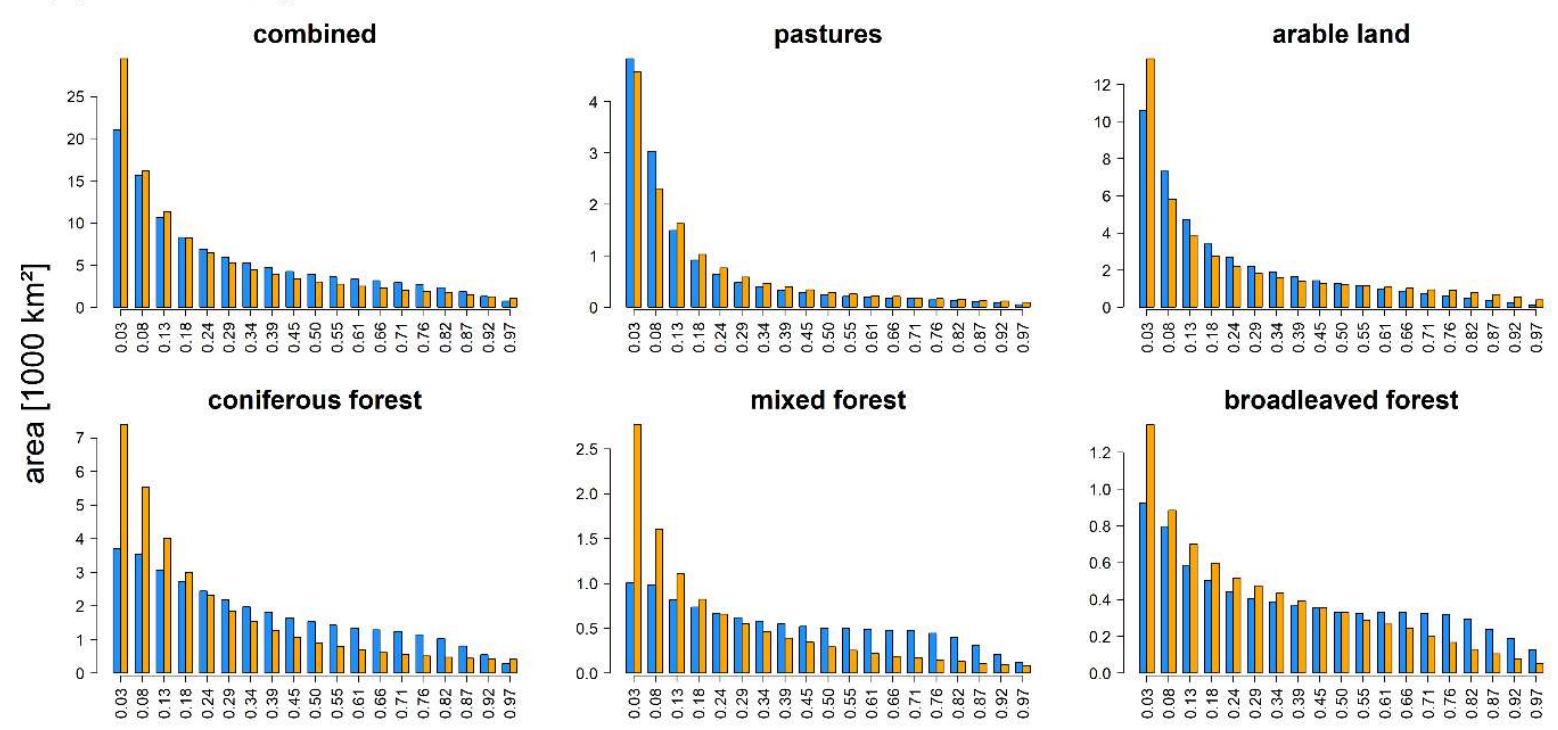

(b) $-2<$ CWB anomaly $<0$
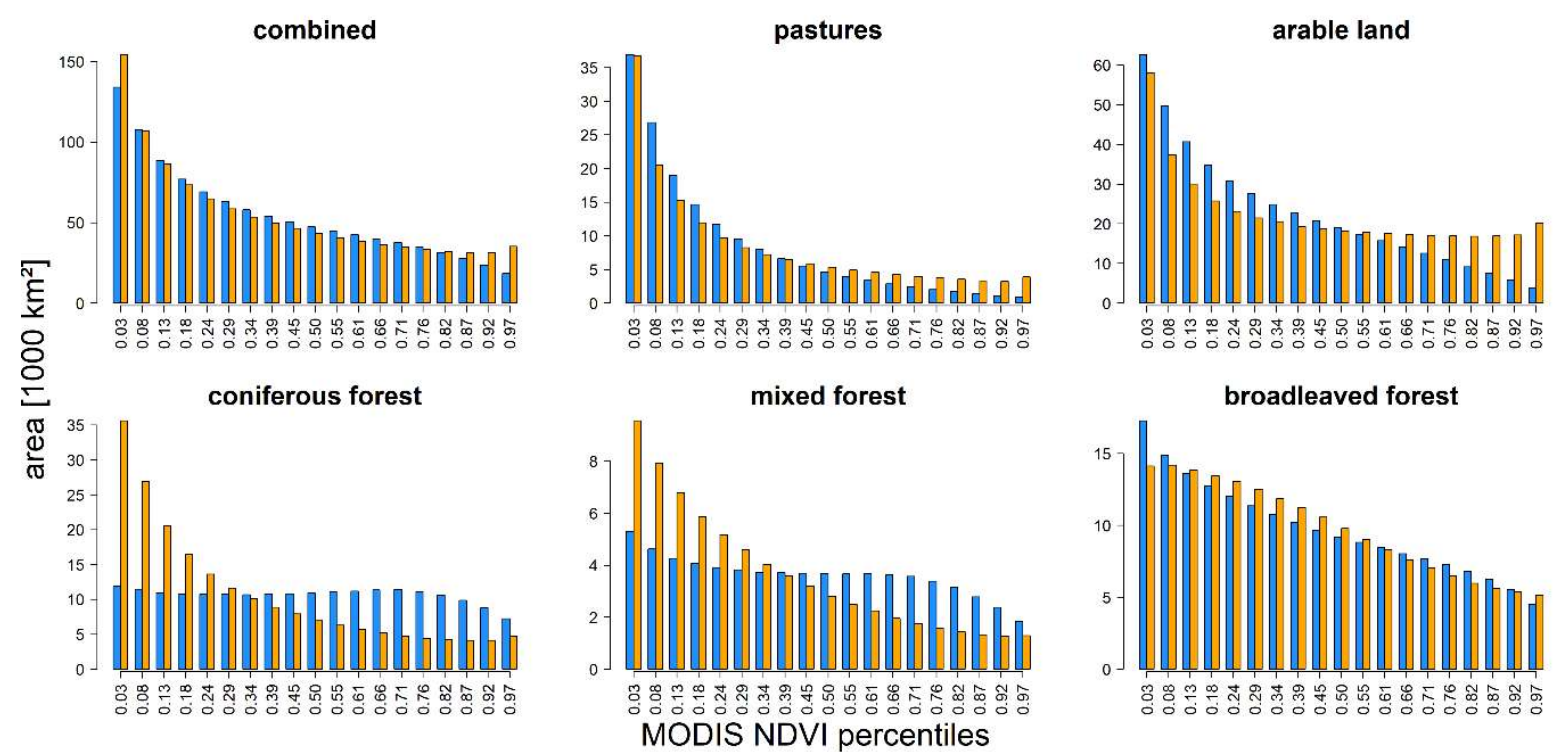

Fig. S12: Histograms depicting the absolute areas (in units of $1000 \mathbf{~ k m}^{2}$ ) representing the nineteen NDVI quantiles pooled according to CORINE land-cover classes for the intersection areas of regions that featured (a) extreme water deficit (CWB-anomaly $<-2)$ and (b) weak water deficit $(-2<$ CWB-anomaly $<0$ ), in both 2003 and 2018 (see Fig. S11 for intersection areas). Blue bars refer to 2003, orange bars to 2018. Histograms depicting the areas for percentiles of EVI are shown in Fig. S13. 
(a) CWB anomaly $<-2$
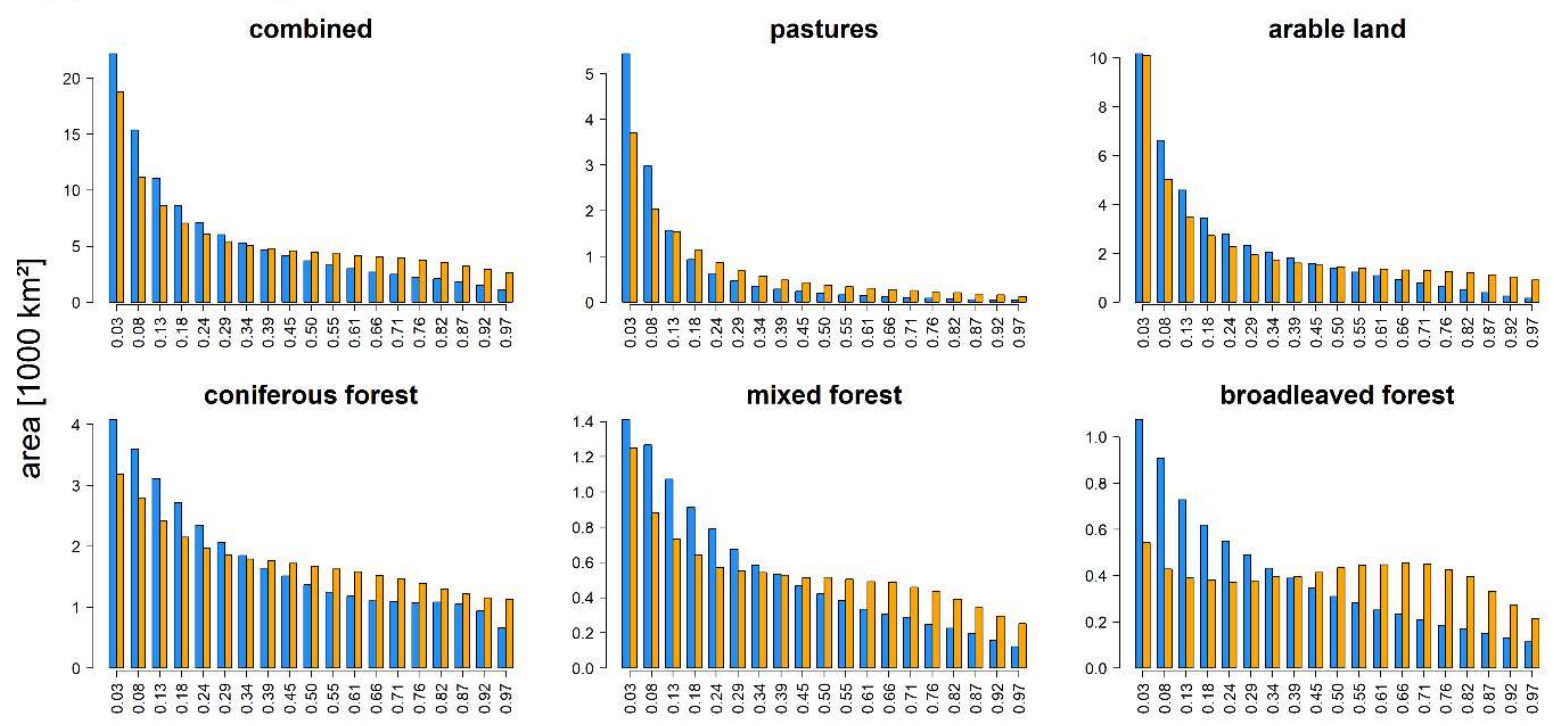

(b) $-2<$ CWB anomaly $<0$
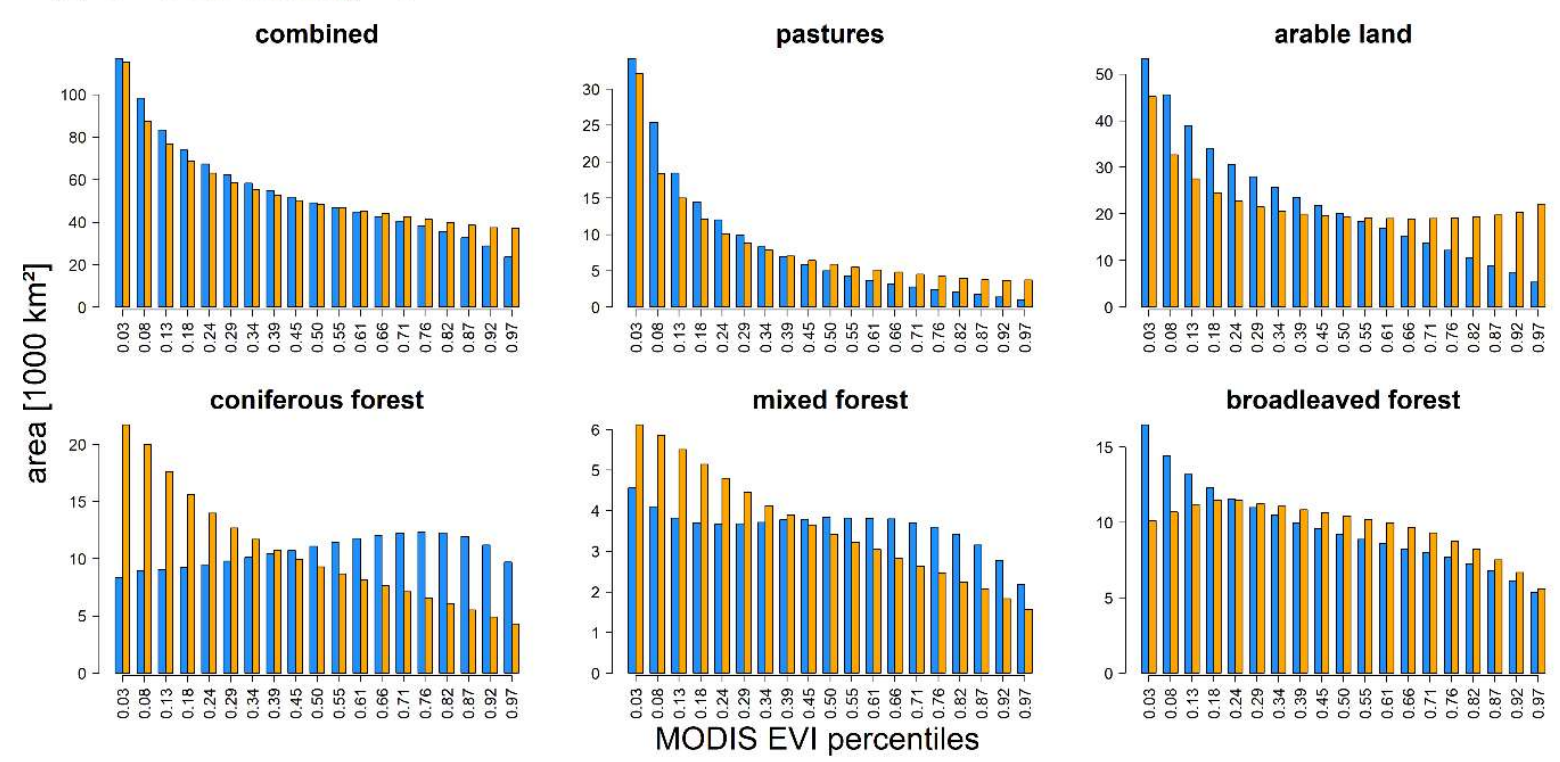

Fig. S13: Histograms depicting the absolute areas (in units of $1000 \mathrm{~km}^{2}$ ) representing the nineteen EVI quantiles pooled according to CORINE land-cover classes for the intersection areas of regions that featured (a) extreme water deficit (CWB-anomaly <-2) and (b) weak water deficit $(-2<$ CWB-anomaly $<0$ ), in both 2003 and 2018 (see Fig. S11 for intersection areas). Blue bars refer to 2003, orange bars to 2018. 
combined

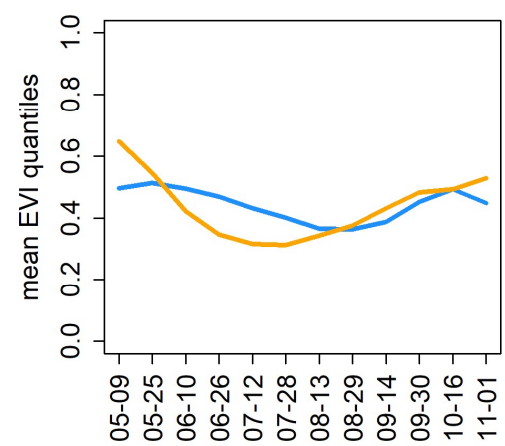

CF

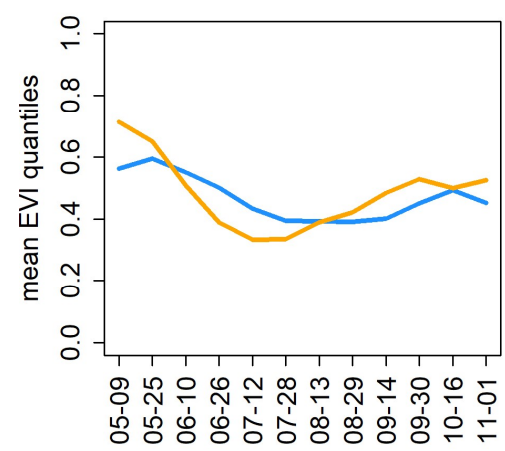

PS

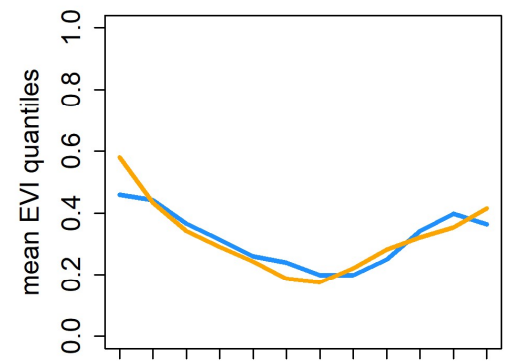

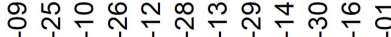

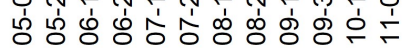

MF

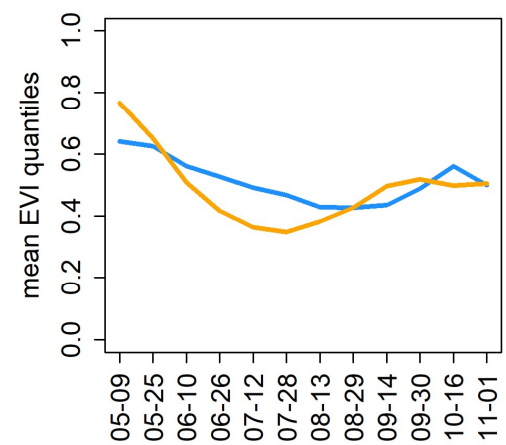

AL

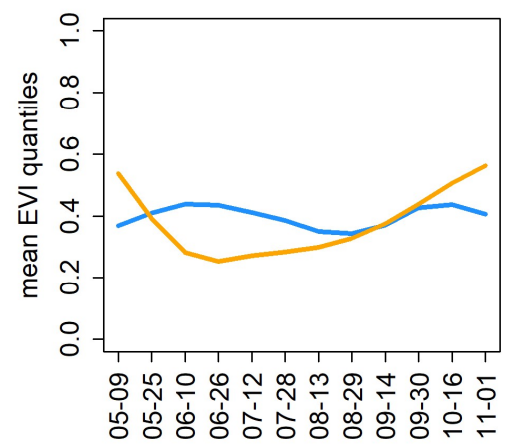

BF

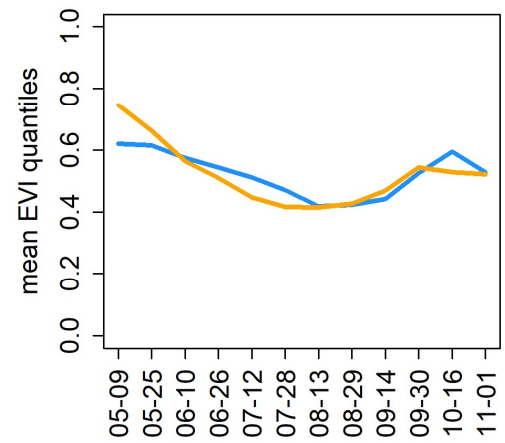

Fig. S14: Time-series of EVI-quantiles averaged over the regions featuring CWB <-2 in August 2003 (blue) and July 2018 (orange) for the five different land-cover classes (b-f) and a combination of those (a). PS = pastures, $\mathrm{AL}=$ arable land, $\mathrm{CF}=$ coniferous forest, $\mathrm{MF}=\operatorname{mixed}$ forest, $\mathrm{BF}=$ broadleaved forest. 
(a)

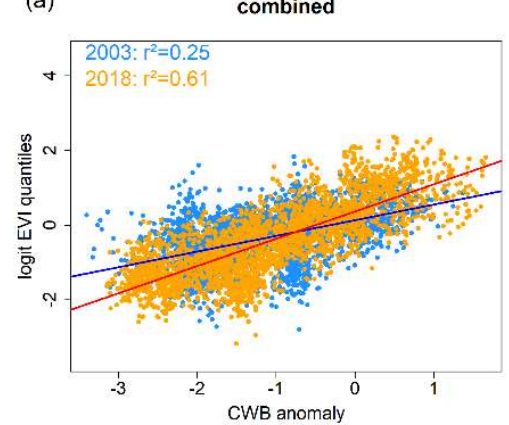

(d)

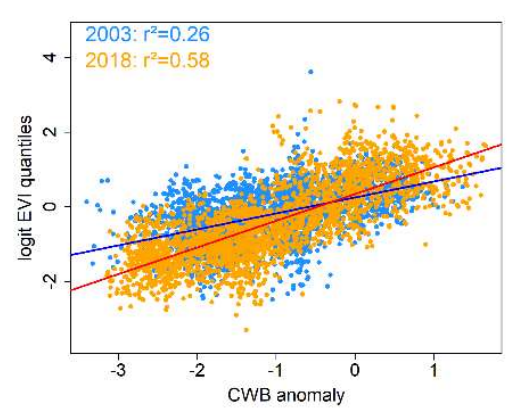

(b)

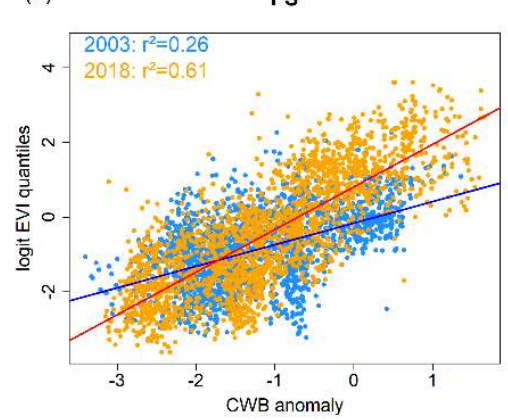

(e)

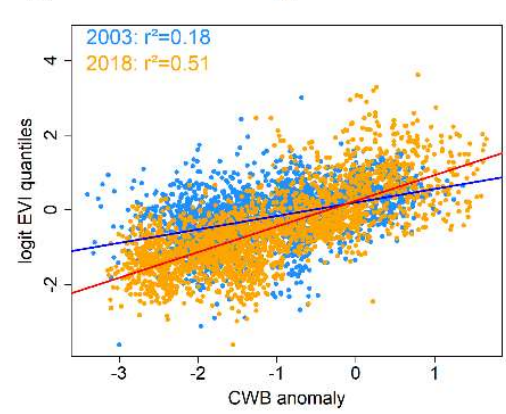

(c) $\mathrm{AL}$

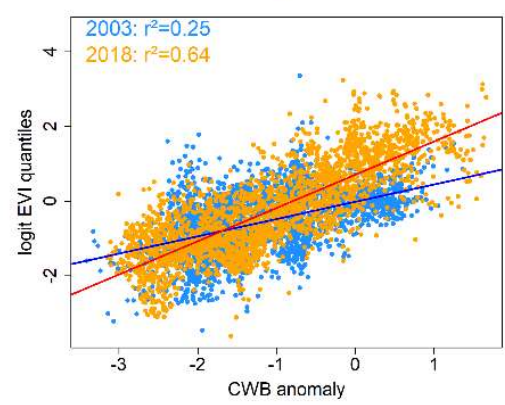

(f)

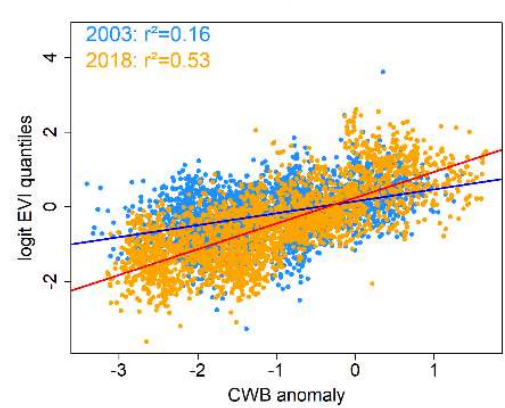

(g)

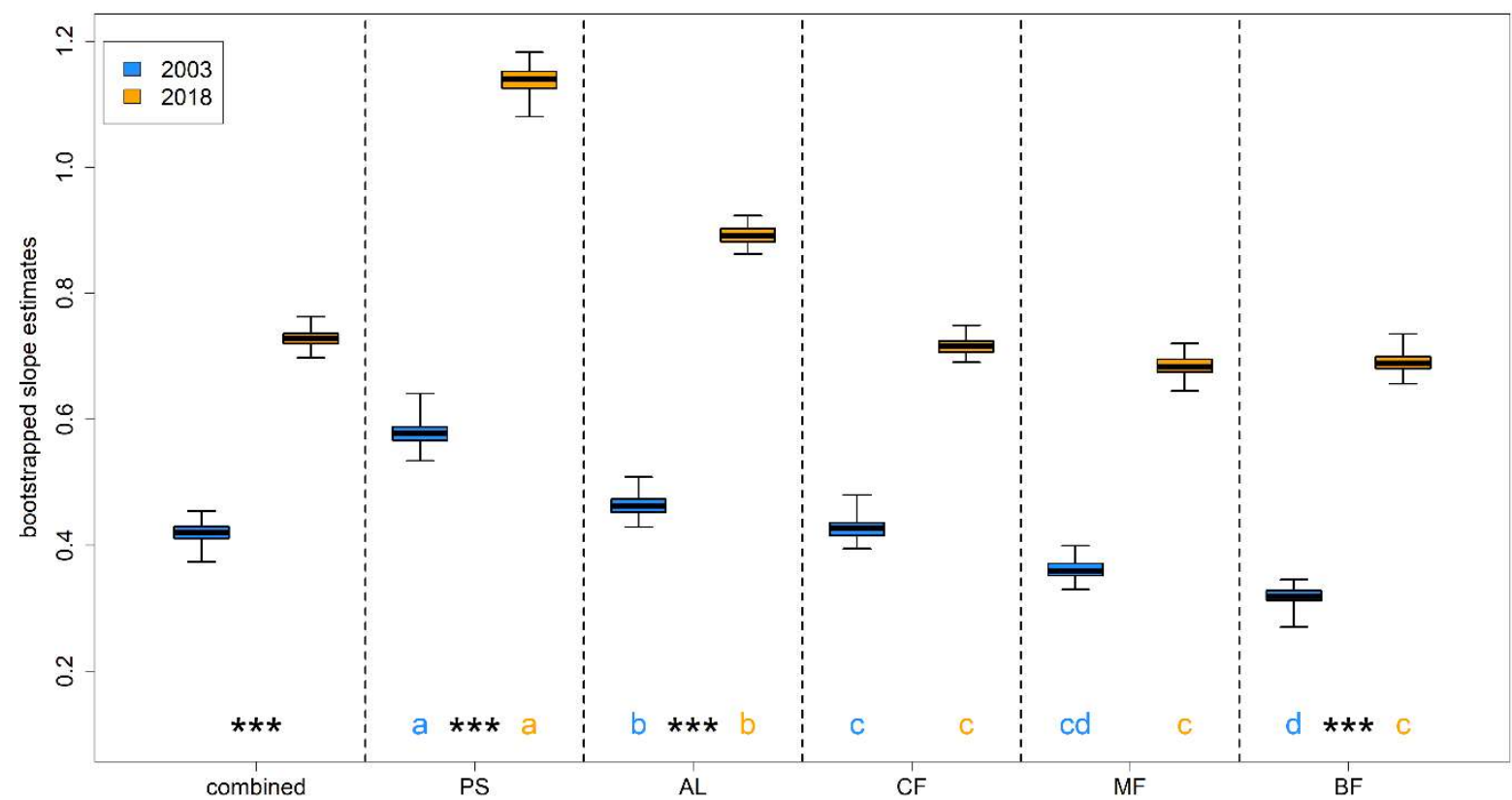

Figure S15: (a-f) Scatterplots depicting the relationship between average logit-transformed EVI-quantiles and mean CWB in 2003 (blue) and 2018 (orange) for pastures (b), arable land (c), coniferous forests (d), mixed forest (e), broadleaved forest (f) and a combination of those (a). Blue lines depict the regression line for 2003, red lines for 2018. (g) Bootstrapped regression slope estimates for the five different land-cover classes as well as their combination. Minor case letters refer to group assignment of land-cover classes according to the overlap of $99.9 \%$ confidence intervals of bootstrapped slopes in 2003 (blue) and 2018 (orange). Significance stars $(* * *)$ indicate no overlap between $99.9 \%$ confidence intervals of 2003 and 2018 for the respective land-cover class. $\mathrm{PS}=$ pastures, $\mathrm{AL}=$ arable land, $\mathrm{CF}=$ coniferous forest, $\mathrm{MF}=$ mixed forest, $\mathbf{B F}=$ broadleaved forest. 
(a) 2003

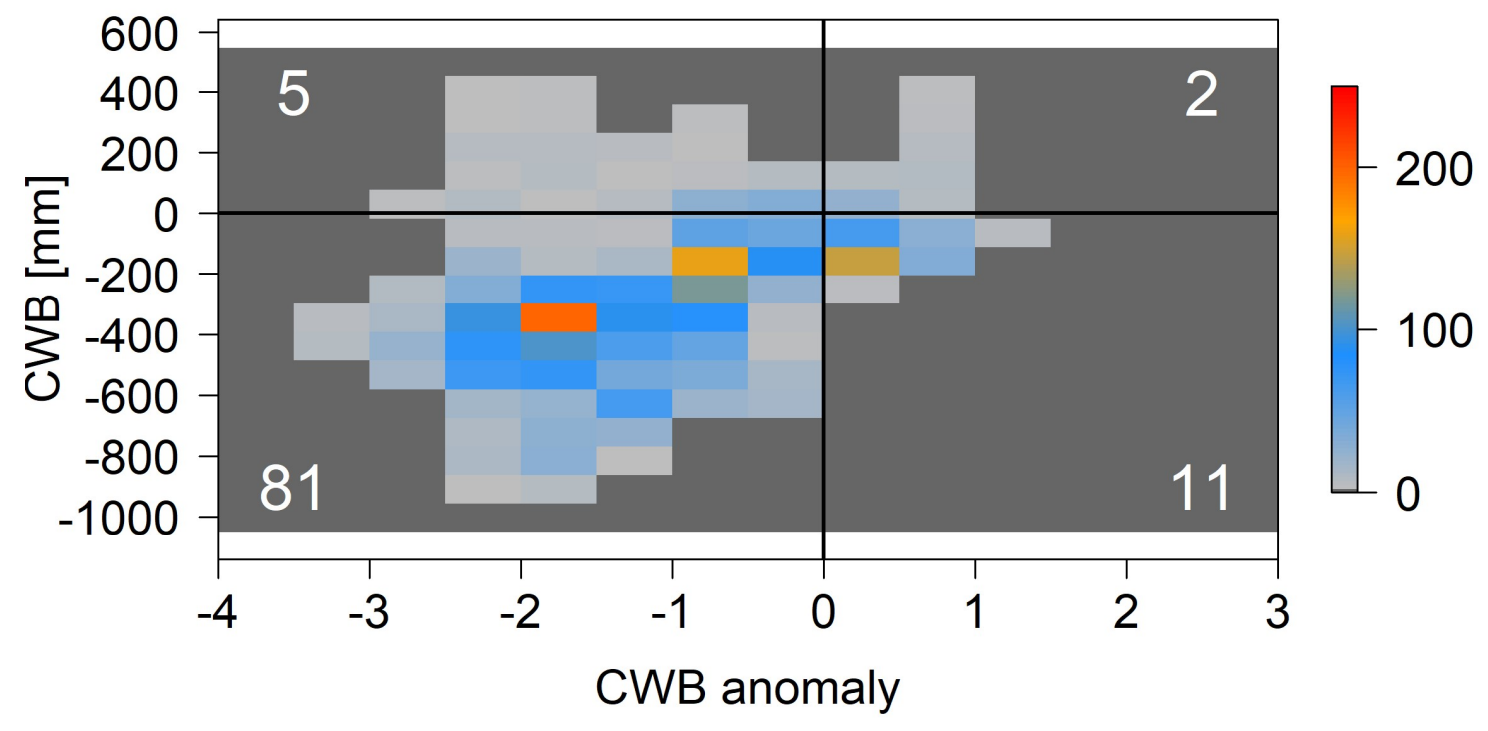

(b) $\quad 2018$

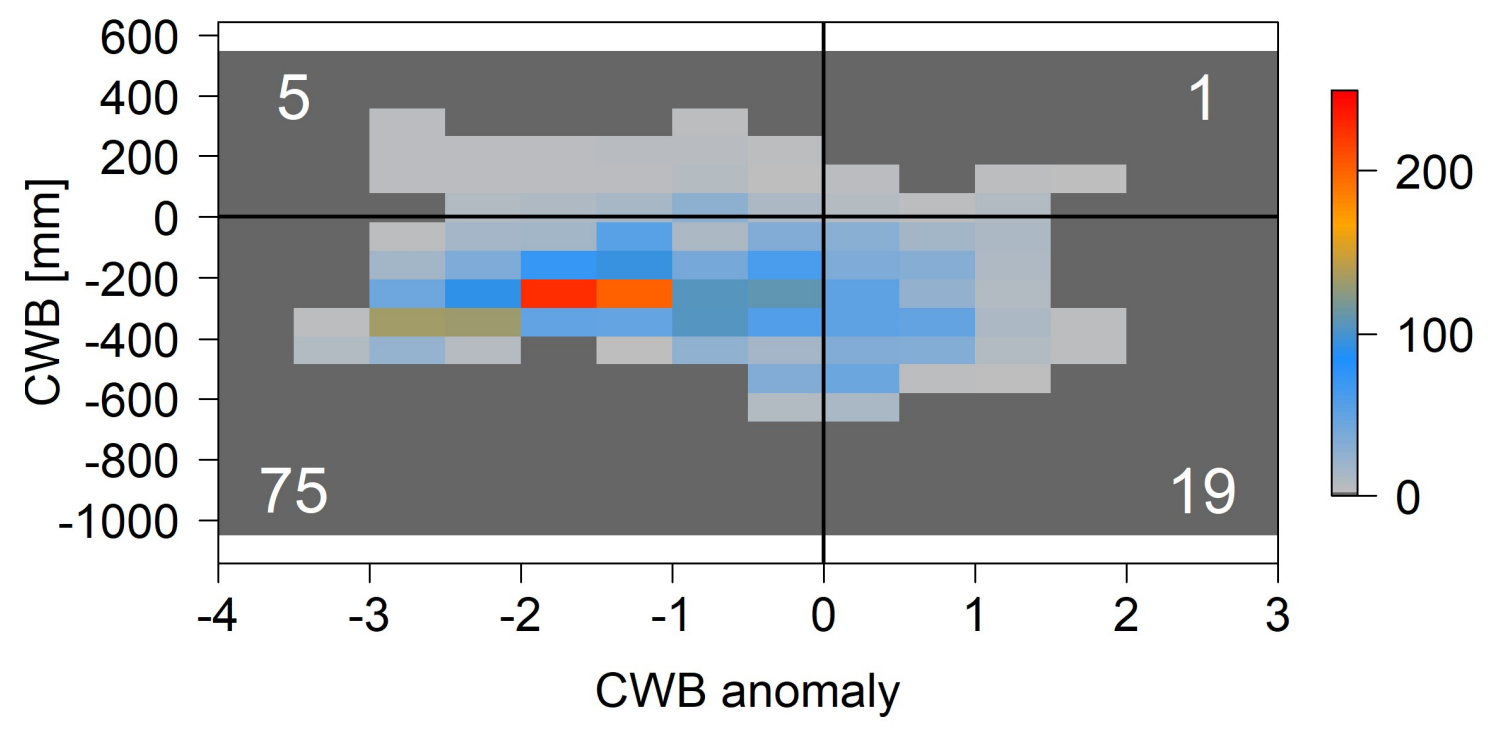

Fig. S16: Comparison of August 2018 (a) and July 2018 (b) CWB anomalies and raw CWB values to indicate absolute amount (colored pixels) as well as the share in percent (values in white) of pixels that featured positive CWB, while CWB anomaly was negative. In both years, 5 (1) percent featured positive CWB for CWB anomalies lower than $0(-2)$. 


\section{Leaf coloration beech $(\mathrm{BBCH}=31)$}

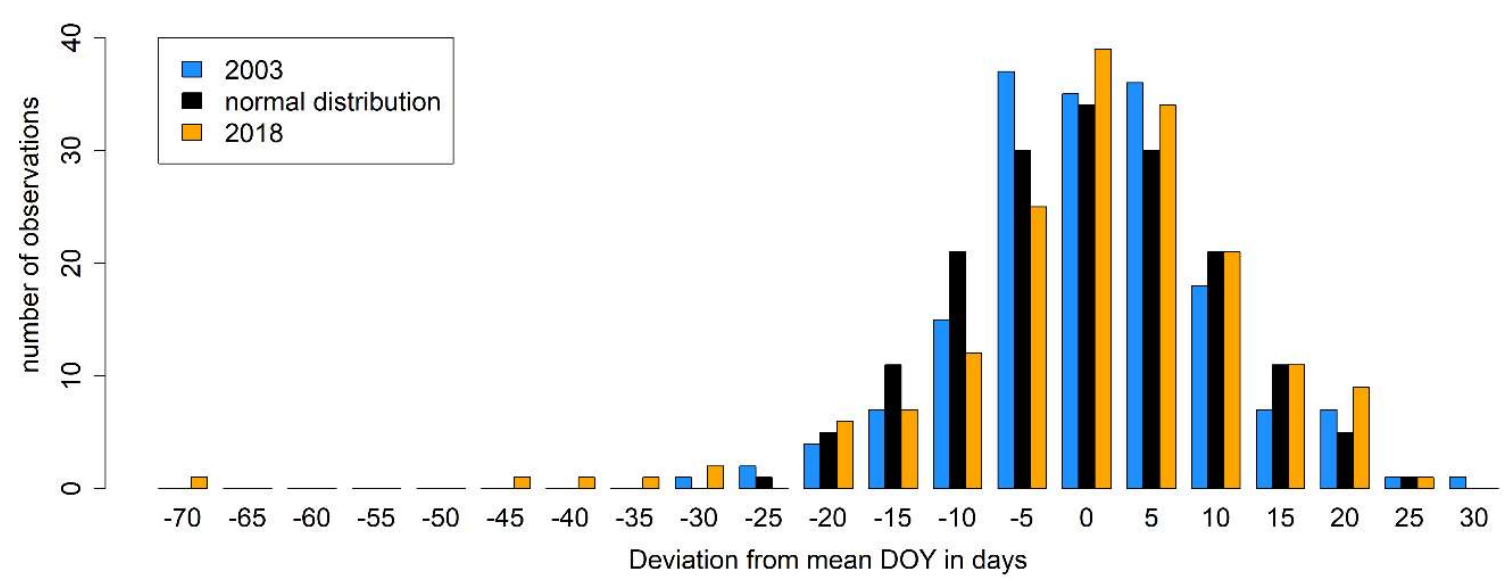

Fig. S17: Deviation of leaf coloration (BBCH code 31) for European beech (Fagus sylvatica) based on phenological observations provided by the German meteorological service (DWD) for 2003 (blue) and 2018 (orange) in comparison to a normal distribution. For each observational point its deviation to the long-term mean was computed. For instance, one observational point reported a 70 days earlier leaf coloration in 2018. 